\title{
FLUKA Monte Carlo Simulations about Cosmic Rays Interactions with Kaidun Meteorite
}

\author{
Turgay Korkut \\ Faculty of Science and Art, Department of Physics, A ̆grı İbrahim Çeçen University, 04100 A ̆grı, Turkey \\ Correspondence should be addressed to Turgay Korkut; turgaykorkut@hotmail.com
}

Received 19 September 2013; Accepted 20 November 2013

Academic Editor: Mehmet Bektasoglu

Copyright (C) 2013 Turgay Korkut. This is an open access article distributed under the Creative Commons Attribution License, which permits unrestricted use, distribution, and reproduction in any medium, provided the original work is properly cited.

\begin{abstract}
An asteroid called Kaidun fell on December 3, 1980, in Yemen $\left(15^{\circ} 0^{\prime} \mathrm{N}, 48^{\circ} 18^{\prime} \mathrm{E}\right)$. Investigations on this large-sized meteorite are ongoing today. In this paper, interactions between cosmic rays-earth atmosphere and cosmic rays-Kaidun meteorite were modeled using a cosmic ray generator FLUKA Monte Carlo code. Isotope distributions and produced particles were given after these interactions. Also, simulation results were compared for these two types of interactions.
\end{abstract}

\section{Introduction}

Cosmic rays (CRs) are high-energy charged particles, coming from space which voyage at close to the speed of light and hit the Earth's atmosphere from anywhere. Essentially, all of the elements in the periodic table; about $89 \%$ of the nuclei are hydrogen (protons), 10\% helium, and about $1 \%$ heavier elements, generate CRs. These heavier elements (such as carbon, oxygen, magnesium, silicon, and iron) are present in about the same relative abundance as in the solar system, but there are important differences in elemental and isotopic composition that provides information on the origin and history of galactic cosmic rays. For example, there is a significant excess of the rare elements $(\mathrm{Li}, \mathrm{Be}$, and $\mathrm{B})$ produced when heavier CRs such as $\mathrm{C}, \mathrm{N}$, and $\mathrm{O}$ fragment into lighter nuclei during collisions with the interstellar gas. The isotope

${ }^{22} \mathrm{Ne}$ is also excessive, showing that the nucleosynthesis of cosmic rays and solar system material has differed. Electrons constitute about $1 \%$ of galactic cosmic rays. It is not known why electrons are apparently less efficiently accelerated than nuclei.

Kaidun meteorite fell in Yemen $\left(15^{\circ} 0^{\prime} \mathrm{N}, 48^{\circ} 18^{\prime} \mathrm{E}\right)$ on 3 December, 1980. It is in the carbonaceous chondrite meteorite class and its mass is about $2 \mathrm{~kg}$. It holds a special place in the world meteorite collection. It is characterized by an unprecedentedly wide variety of meteorite material in its makeup. It is believed that Kaidun originated from Phobos, one of two Martian moons [1]. Two new minerals called Andreyivanovite and Florenskyite were discovered after research on the meteorite. There are several studies about Kaidun meteorite and its minerals (Andreyivanovite and Florenskyite). These studies are generally focused on the chronology of meteorite [2-6], the geochemistry [7-16], mineralogy [17-19], and the space research [20-25].

FLUKA makes Monte Carlo estimations about particlematter interactions. FLUKA Monte Carlo package has already been used to simulate CRs generation and interactions in several research papers [26-29]. Interactions between CRsKaidun meteorite and CRs-earth atmosphere were simulated in the present paper.

\section{Methodology}

FLUKA is a FORTRAN-based Monte Carlo tool used for calculations of particle transportations and interactions with different materials used in many different application areas such as calorimetry, activation, dosimetry, detector design, accelerators, cosmic rays, neutrino physics, and radiotherapy. In this code, 60 different subatomic particles are described and almost all of the materials used in many applications can be defined using suitable cards [30, 31]. In 2010, FLUKA Monte Carlo code was presented as a new high-energy cosmic ray generator. With this new release, FLUKA is able to simulate interactions between high-energy cosmic rays and Earth's atmosphere. In this code using the combinational 

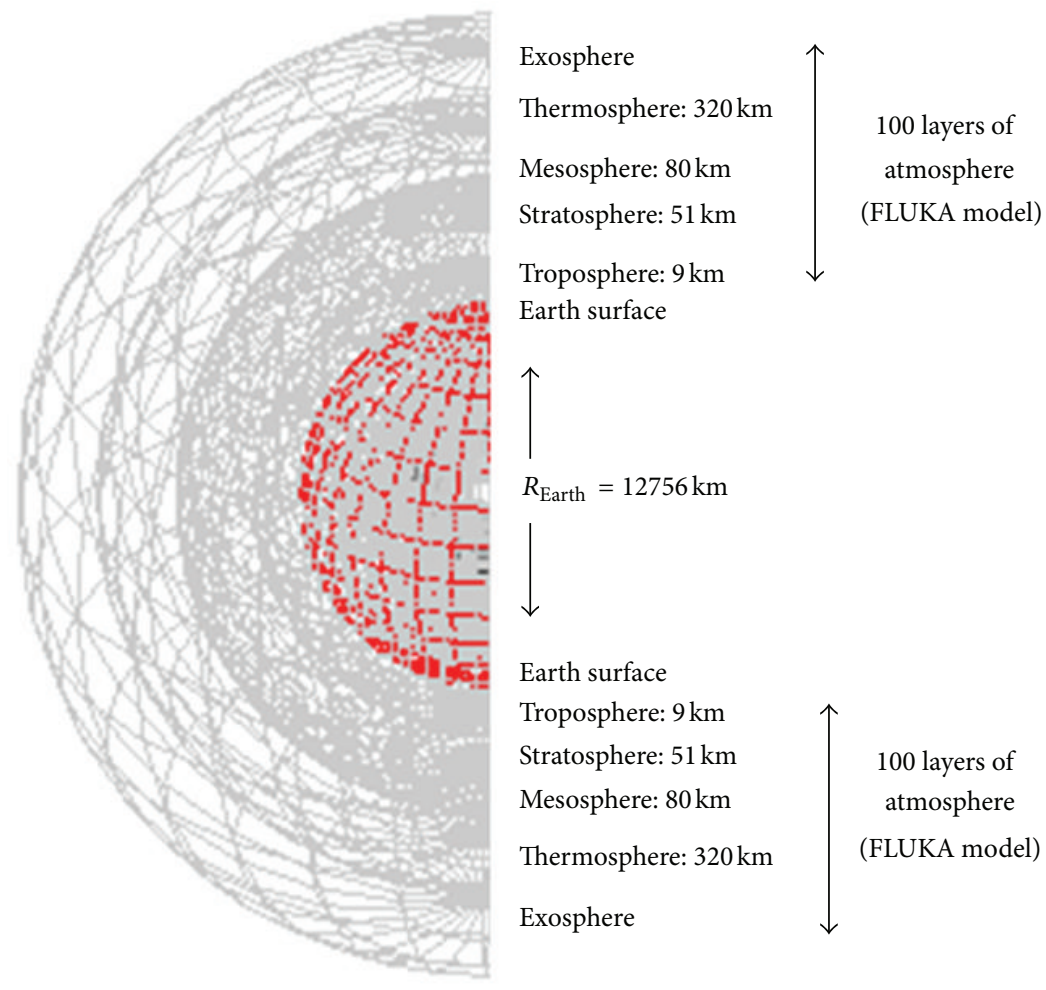

FIGURE 1: 100 layered FLUKA atmosphere model.

geometry package included into the general code, Earth atmosphere is modeled with a set of 100 concentric spherical shells (Figure 1). For the material components of Earth atmosphere, the same material at different pressure and temperature conditions for each layer was used. Composition of Kaidun meteorite was taken in the literature. Hadronic interactions were activated using DPMJET package [32]. As for primary cosmic ray beam primary mass composition model is used such as $90 \%$ of protons and $9 \%$ of alphas [33]. As for scores of simulations, we wanted primary proton fluency, produced radioisotopes, and secondary particles existing after interactions. The general FLUKA code exists on the website at http://www.fluka.org/.

\section{Results}

In terms of performance of FLUKA code about capability of cosmic ray-atmosphere interactions, we plotted primary proton fluency as a function of primary proton energies (Figure 2). As can be seen in this figure, proton energies can reach up to $10 \mathrm{TeV}$ after interactions between CRs and Earth's atmosphere.

In Table 1, radioisotopes produced after interactions between CRs and Earth's atmosphere are seen. 66 different cosmogenic isotopes were produced as atomic number range from 1 to 20. Abundance of ${ }^{4} \mathrm{He},{ }^{14} \mathrm{C}$, and ${ }^{14} \mathrm{~N}$ isotopes is remarkable. Trace isotopes are ${ }^{37} \mathrm{~S},{ }^{18} \mathrm{O}$, and ${ }^{34} \mathrm{P}$.

In Table 2, isotope products derived from CRs-Kaidun meteorite interactions were given. A wide range of isotope

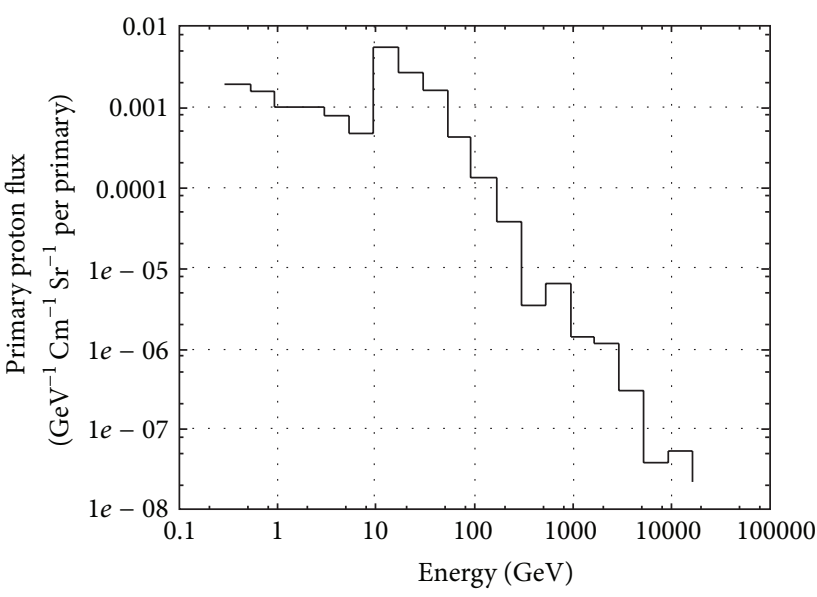

FIGURE 2: The differential primary proton fluence spectra.

distribution (atomic number from 1 to 79 ) is seen unlike Table 1. In these products, the most abundance is ${ }^{56} \mathrm{Fe}$ because of Kaidun's high Fe content. Other important isotopes are ${ }^{55} \mathrm{Fe},{ }^{55} \mathrm{Mn}$, and ${ }^{54} \mathrm{Mn}$. Small amounts of ${ }^{49} \mathrm{Ca},{ }^{82} \mathrm{Br}$, and ${ }^{108} \mathrm{Ag}$ are also produced.

Table 3 shows number of particles produced after CRsatmosphere and CRs-Kaidun. In this table, neutrons and protons are outstanding. Antisigma (+), antisigma (-), xi (0), anti-xi (0), and anti-xi (+) are trace particles. Looking at the relative differences, it is seen that the biggest changes occur 
TABLE 1: Isotope distribution after interactions between CR and Earth's atmosphere.

\begin{tabular}{|c|c|}
\hline Isotope & Number of nuclei \\
\hline $\mathrm{H}-1$ & $4,63 E+02$ \\
\hline $\mathrm{H}-2$ & $6,84 E+01$ \\
\hline $\mathrm{H}-3$ & $2,24 E+01$ \\
\hline $\mathrm{He}-3$ & $2,12 E+01$ \\
\hline $\mathrm{He}-4$ & $4,62 E+02$ \\
\hline He-6 & $4,53 E-02$ \\
\hline $\mathrm{Li}-6$ & $2,27 E+01$ \\
\hline $\mathrm{Li}-7$ & $1,60 E+01$ \\
\hline $\mathrm{Be}-7$ & $6,78 E+00$ \\
\hline He-8 & $3,25 E-04$ \\
\hline $\mathrm{Li}-8$ & $3,51 E+00$ \\
\hline B-8 & $1,10 E-01$ \\
\hline Li-9 & $6,16 E-03$ \\
\hline Be-9 & $2,10 E+01$ \\
\hline C-9 & $4,01 E-02$ \\
\hline Be-10 & $1,49 E+01$ \\
\hline B-10 & $1,99 E+01$ \\
\hline $\mathrm{C}-10$ & $1,03 E+00$ \\
\hline Be-11 & $2,83 E-01$ \\
\hline B-11 & $6,93 E+01$ \\
\hline C-11 & $1,22 E+01$ \\
\hline B-12 & $3,64 E+00$ \\
\hline C-12 & $7,27 E+01$ \\
\hline $\mathrm{N}-12$ & $3,11 E-04$ \\
\hline B-13 & $5,01 E-01$ \\
\hline C-13 & $9,50 E+01$ \\
\hline $\mathrm{N}-13$ & $1,73 E+01$ \\
\hline C-14 & $1,10 E+02$ \\
\hline $\mathrm{N}-14$ & $2,19 E+02$ \\
\hline $\mathrm{O}-14$ & $3,97 E-01$ \\
\hline C-15 & $7,48 E-04$ \\
\hline N-15 & $2,40 E+01$ \\
\hline O-15 & $8,70 E+00$ \\
\hline N-16 & $7,91 E-01$ \\
\hline O-16 & $5,42 E+01$ \\
\hline $\mathrm{O}-17$ & $1,99 E-03$ \\
\hline O-18 & $6,53 E-05$ \\
\hline $\mathrm{Ne}-20$ & $1,25 E-04$ \\
\hline $\mathrm{Ne}-21$ & $9,71 E-01$ \\
\hline $\mathrm{Na}-22$ & $1,53 E-04$ \\
\hline $\mathrm{Al}-28$ & $2,52 E-01$ \\
\hline $\mathrm{Si}-28$ & $1,81 E-01$ \\
\hline $\mathrm{Si}-30$ & $3,50 E-04$ \\
\hline Si-31 & $4,01 E-01$ \\
\hline S-32 & $3,22 E-04$ \\
\hline P-33 & $8,92 E-04$ \\
\hline S-33 & $1,86 E-02$ \\
\hline P-34 & $7,41 E-05$ \\
\hline S-34 & $5,15 E-03$ \\
\hline S-35 & $5,42 E-02$ \\
\hline
\end{tabular}

TABLE 1: Continued.

\begin{tabular}{lc}
\hline Isotope & Number of nuclei \\
\hline Cl-35 & $1,42 E-03$ \\
S-36 & $6,77 E-02$ \\
Cl-36 & $1,13 E-02$ \\
Ar-36 & $9,21 E-05$ \\
S-37 & $5,74 E-05$ \\
Cl-37 & $1,64 E-02$ \\
Ar-37 & $4,41 E-02$ \\
Cl-38 & $7,48 E-02$ \\
Ar-38 & $1,72 E+00$ \\
Cl-39 & $5,60 E-03$ \\
Ar-39 & $8,78 E-01$ \\
Cl-40 & $5,99 E-04$ \\
Ar-40 & $1,32 E+00$ \\
K-40 & $1,10 E-01$ \\
Ar-41 & $1,36 E-01$ \\
Ca-42 & $2,44 E-04$ \\
\hline
\end{tabular}

for xi (0), sigma (-), and antilambda. The amount of ${ }^{3} \mathrm{He}$ has not changed.

In Table 4, numbers of secondary particles generated in inelastic interactions were given. 47 different particles for atmosphere and 50 for Kaidun meteorite were detected by simulations. After each two interactions with CRs, produced maximum particle is neutrons as up to three millions. Also, a small amount of $\mathrm{D}$ mesons $(\mathrm{D}+)$ was produced. Judging by the relative differences, maximum changes occur for $\mathrm{D}$ meson (D+), delta baryon (D0BAR), and omega baryon. Charmed omega baryons (OMEGAC), tau neutrinos (NEUTRIT), and their antiparticles (ANEUTRIT) were formed only after CRs-Kaidun interactions.

Finally in Table 5, numbers of decay products have been shown. 26 different decay products for atmosphere and 31 of Kaidun interactions were detected. In these products, there are many photons, electrons, positrons, muons, and neutrinos (electron and tau). Relative difference of anti-xi $(+)$, $\mathrm{xi}(0)$, and negative kaon particles is higher than that of other products.

\section{Conclusions}

Recently, the interest in dark matter investigations, Mars mission studies, and actual cosmological studies is increasing rapidly. On these issues, meteorites, cosmic dust, and cosmogenic subatomic particles from outer space give some hints for researchers. In this paper, we simulated CRs interactions with Earth's atmosphere and Kaidun meteorite by FLUKA code which is a popular CR generator. For this aim, Earth's atmosphere, Kaidun sample, and primary cosmic rays consisting of protons, alphas, and a small amount of other particles were modeled. As for outputs of simulations, produced isotopes and subatomic particles by atmosphere and Kaidun were obtained. Looking at the isotope distributions, 185 types of isotopes were produced after CRs-Kaidun 
TABLE 2: Isotope distribution after interactions between CR and Kaidun meteorite.

\begin{tabular}{|c|c|}
\hline Isotope & Number of nuclei \\
\hline $\mathrm{H}-1$ & $2,89 E+01$ \\
\hline $\mathrm{H}-2$ & $1,41 E+00$ \\
\hline $\mathrm{H}-3$ & $3,34 E-01$ \\
\hline $\mathrm{He}-3$ & $5,62 E-02$ \\
\hline $\mathrm{He}-4$ & $5,41 E+00$ \\
\hline $\mathrm{He}-6$ & $4,09 E-02$ \\
\hline Li-6 & $2,37 E-04$ \\
\hline $\mathrm{Li}-7$ & $3,73 E-04$ \\
\hline $\mathrm{Be}-7$ & $1,85 E-05$ \\
\hline Li-9 & $2,43 E-06$ \\
\hline Be-9 & $4,22 E-06$ \\
\hline B-10 & $8,91 E-06$ \\
\hline B-11 & $2,66 E-04$ \\
\hline C-11 & $4,70 E-04$ \\
\hline B-12 & $2,70 E-05$ \\
\hline $\mathrm{C}-12$ & $5,41 E-04$ \\
\hline $\mathrm{C}-13$ & $2,60 E-04$ \\
\hline $\mathrm{N}-13$ & $3,94 E-06$ \\
\hline C-14 & $1,09 E-02$ \\
\hline $\mathrm{N}-14$ & $2,16 E-03$ \\
\hline C-15 & $1,09 E-05$ \\
\hline $\mathrm{N}-15$ & $8,53 E-02$ \\
\hline O-15 & $1,20 E-05$ \\
\hline $\mathrm{N}-16$ & $3,86 E-05$ \\
\hline O-16 & $5,72 E-02$ \\
\hline O-17 & $6,20 E-02$ \\
\hline F-17 & $3,64 E-05$ \\
\hline $\mathrm{O}-18$ & $2,04 E-02$ \\
\hline F-18 & $8,53 E-04$ \\
\hline F-19 & $1,58 E-02$ \\
\hline $\mathrm{O}-20$ & $3,72 E-06$ \\
\hline F-20 & $1,30 E-03$ \\
\hline $\mathrm{Ne}-20$ & $2,39 E-02$ \\
\hline F-21 & $2,99 E-04$ \\
\hline $\mathrm{Ne}-21$ & $3,12 E-01$ \\
\hline $\mathrm{Ne}-22$ & $4,22 E-01$ \\
\hline $\mathrm{Ne}-23$ & $2,40 E-02$ \\
\hline $\mathrm{Na}-21$ & $5,72 E-05$ \\
\hline $\mathrm{Na}-22$ & $8,06 E-02$ \\
\hline $\mathrm{Na}-23$ & $2,09 E+00$ \\
\hline Mg-23 & $6,04 E-06$ \\
\hline $\mathrm{Na}-24$ & $3,09 E-02$ \\
\hline $\mathrm{Mg}-24$ & $1,99 E-04$ \\
\hline Al-26 & $4,17 E-05$ \\
\hline Mg-27 & $4,05 E-08$ \\
\hline $\mathrm{Al}-27$ & $2,21 E-02$ \\
\hline $\mathrm{Al}-28$ & $1,24 E-03$ \\
\hline $\mathrm{Si}-28$ & $5,30 E-03$ \\
\hline Al-29 & $1,30 E-05$ \\
\hline Si-29 & $5,07 E-03$ \\
\hline Al-30 & $3,38 E-03$ \\
\hline Si-30 & $6,41 E-02$ \\
\hline P-30 & $5,35 E-04$ \\
\hline
\end{tabular}

TABLE 2: Continued.

\begin{tabular}{|c|c|}
\hline Isotope & Number of nuclei \\
\hline Si-31 & $2,43 E-06$ \\
\hline P-31 & $2,05 E-02$ \\
\hline P-32 & $4,95 E-02$ \\
\hline S-32 & $1,06 E-01$ \\
\hline P-33 & $1,66 E-04$ \\
\hline S-33 & $6,57 E-02$ \\
\hline S-34 & $1,07 E-01$ \\
\hline $\mathrm{Cl}-34$ & $1,18 E-03$ \\
\hline S-35 & $3,28 E-05$ \\
\hline $\mathrm{Cl}-35$ & $5,72 E-02$ \\
\hline S-36 & $3,15 E-04$ \\
\hline $\mathrm{Cl}-36$ & $8,91 E-02$ \\
\hline Ar-36 & $7,90 E-02$ \\
\hline $\mathrm{Cl}-37$ & $6,31 E-02$ \\
\hline Ar-37 & $3,54 E-01$ \\
\hline $\mathrm{K}-37$ & $4,75 E-06$ \\
\hline $\mathrm{Cl}-38$ & $1,30 E-04$ \\
\hline Ar-38 & $1,20 E-01$ \\
\hline $\mathrm{K}-38$ & $5,57 E-03$ \\
\hline $\mathrm{Ca}-38$ & $4,01 E-06$ \\
\hline Cl-39 & $1,22 E-03$ \\
\hline Ar-39 & $2,31 E-02$ \\
\hline K-39 & $3,78 E-01$ \\
\hline $\mathrm{Ca}-39$ & $3,47 E-02$ \\
\hline $\mathrm{Cl}-40$ & $4,40 E-06$ \\
\hline Ar- 40 & $5,99 E-05$ \\
\hline $\mathrm{K}-40$ & $4,53 E-01$ \\
\hline $\mathrm{Ca}-40$ & $3,61 E-01$ \\
\hline K-41 & $1,13 E-01$ \\
\hline $\mathrm{Ca}-41$ & $1,57 E-02$ \\
\hline Sc-41 & $1,51 E-05$ \\
\hline $\mathrm{K}-42$ & $1,16 E-03$ \\
\hline $\mathrm{Ca}-42$ & $8,91 E-02$ \\
\hline Sc- 42 & $9,01 E-05$ \\
\hline $\mathrm{K}-43$ & $1,08 E-04$ \\
\hline $\mathrm{Ca}-43$ & $2,50 E-02$ \\
\hline Sc- 43 & $4,28 E-04$ \\
\hline $\mathrm{Ca}-44$ & $5,46 E-02$ \\
\hline Sc- 44 & $8,85 E-02$ \\
\hline $\mathrm{Ca}-45$ & $1,22 E-03$ \\
\hline Sc- 45 & $5,30 E-02$ \\
\hline $\mathrm{Ca}-46$ & $8,53 E-06$ \\
\hline Sc-46 & $2,51 E-02$ \\
\hline $\mathrm{Ca}-47$ & $4,37 E-04$ \\
\hline Sc- 47 & $1,28 E-03$ \\
\hline Sc- 48 & $6,73 E-05$ \\
\hline $\mathrm{Ca}-49$ & $5,88 E-09$ \\
\hline Sc- 49 & $1,17 E-05$ \\
\hline $\mathrm{Ti}-44$ & $3,27 E-03$ \\
\hline $\mathrm{Ti}-45$ & $2,85 E-02$ \\
\hline $\mathrm{Ti}-46$ & $3,60 E-01$ \\
\hline V-46 & $7,42 E-06$ \\
\hline $\mathrm{Ti}-47$ & $3,41 E-01$ \\
\hline $\mathrm{V}-47$ & $2,82 E-03$ \\
\hline
\end{tabular}


TABLE 2: Continued.

\begin{tabular}{|c|c|}
\hline Isotope & Number of nuclei \\
\hline $\mathrm{Ti}-48$ & $2,99 E-01$ \\
\hline $\mathrm{V}-48$ & $2,24 E-01$ \\
\hline $\mathrm{Cr}-48$ & $5,46 E-02$ \\
\hline Ti-49 & $6,89 E-02$ \\
\hline$V-49$ & $5,99 E-01$ \\
\hline $\mathrm{Cr}-49$ & $1,25 E-01$ \\
\hline Ti-50 & $7,63 E-02$ \\
\hline V-50 & $6,63 E-01$ \\
\hline Cr-50 & $7,95 E-01$ \\
\hline Mn-50 & $1,23 E-01$ \\
\hline$V-51$ & $2,11 E-01$ \\
\hline Cr-51 & $8,80 E-01$ \\
\hline $\mathrm{Mn}-51$ & $3,54 E-01$ \\
\hline$V-52$ & $8,06 E-02$ \\
\hline Cr-52 & $1,72 E+00$ \\
\hline $\mathrm{Mn}-52$ & $6,52 E-01$ \\
\hline $\mathrm{Fe}-52$ & $8,80 E-02$ \\
\hline$V-53$ & $1,26 E-05$ \\
\hline Cr-53 & $7,00 E-01$ \\
\hline Mn-53 & $1,58 E+00$ \\
\hline $\mathrm{Fe}-53$ & $4,64 E-01$ \\
\hline Cr-54 & $2,06 E-01$ \\
\hline Mn-54 & $2,83 E+00$ \\
\hline Fe-54 & $2,48 E+00$ \\
\hline Co-54 & $1,11 E-05$ \\
\hline Cr-55 & $1,13 E-02$ \\
\hline Mn-55 & $3,07 E+00$ \\
\hline Fe-55 & $3,75 E+00$ \\
\hline Co-55 & $1,23 E-01$ \\
\hline Mn-56 & $1,02 E+00$ \\
\hline Fe-56 & $2,46 E+01$ \\
\hline Co-56 & $2,03 E-01$ \\
\hline $\mathrm{Ni}-56$ & $3,50 E-05$ \\
\hline Mn-57 & $3,11 E-03$ \\
\hline $\mathrm{Fe}-57$ & $2,60 E+00$ \\
\hline Co-57 & $2,88 E-01$ \\
\hline $\mathrm{Ni}-57$ & $7,05 E-02$ \\
\hline Mn-58 & $5,72 E-06$ \\
\hline $\mathrm{Fe}-58$ & $7,74 E-02$ \\
\hline Co-58 & $3,33 E-01$ \\
\hline $\mathrm{Ni}-58$ & $7,32 E-01$ \\
\hline Fe-59 & $2,25 E-03$ \\
\hline Co-59 & $9,75 E-02$ \\
\hline $\mathrm{Ni}-59$ & $2,91 E-01$ \\
\hline Co-60 & $2,43 E-01$ \\
\hline $\mathrm{Ni}-60$ & $5,41 E-01$ \\
\hline $\mathrm{Cu}-60$ & $5,08 E-06$ \\
\hline Co-61 & $2,13 E-03$ \\
\hline $\mathrm{Ni}-61$ & $7,32 E-02$ \\
\hline $\mathrm{Cu}-61$ & $2,34 E-02$ \\
\hline Co-62 & $2,34 E-02$ \\
\hline $\mathrm{Ni}-62$ & $2,90 E-02$ \\
\hline $\mathrm{Ni}-63$ & $2,93 E-02$ \\
\hline $\mathrm{Cu}-63$ & $2,85 E-04$ \\
\hline
\end{tabular}

TABLE 2: Continued.

\begin{tabular}{lc}
\hline Isotope & Number of nuclei \\
\hline Ni-64 & $2,49 E-02$ \\
Cu-64 & $6,52 E-06$ \\
Zn-64 & $2,98 E-04$ \\
Ni-65 & $1,08 E-04$ \\
Cu-65 & $2,99 E-04$ \\
Zn-65 & $5,99 E-04$ \\
Zn-66 & $5,94 E-04$ \\
Zn-67 & $8,53 E-04$ \\
Zn-68 & $1,81 E-03$ \\
Zn-69 & $2,60 E-04$ \\
Ga-69 & $5,62 E-06$ \\
Ga-72 & $2,75 E-05$ \\
As-74 & $2,82 E-04$ \\
As-75 & $4,13 E-06$ \\
As-76 & $4,62 E-05$ \\
Br-82 & $7,74 E-07$ \\
Kr-83 & $6,89 E-05$ \\
Kr-84 & $1,85 E-04$ \\
Rb-84 & $8,69 E-02$ \\
Ag-108 & $8,11 E-07$ \\
Ag-110 & $2,16 E-05$ \\
Cd-114 & $3,57 E-05$ \\
Ir-192 & $5,09 E-06$ \\
Au-197 & $2,33 E-06$ \\
\hline
\end{tabular}

TABLE 3: Number of particles generated by interactions.

\begin{tabular}{lccc}
\hline Particle name & Atmosphere & Kaidun & $\begin{array}{c}\text { Relative } \\
\text { difference }(\%)\end{array}$ \\
\hline 4-HELIUM & $8,84 E+03$ & $9,03 E+03$ & 2,13 \\
3-HELIUM & $1,60 E+03$ & $1,60 E+03$ & - \\
TRITON & $2,13 E+03$ & $2,14 E+03$ & 0,47 \\
DEUTERON & $3,20 E+03$ & $3,26 E+03$ & 1,86 \\
HEAVYION & $1,03 E+03$ & $1,09 E+03$ & 5,66 \\
PROTON & $4,57 E+05$ & $4,61 E+05$ & 0,87 \\
APROTON & $1,56 E+03$ & $1,60 E+03$ & 2,53 \\
NEUTRON & $1,67 E+06$ & $1,69 E+06$ & 1,19 \\
ANEUTRON & $1,55 E+03$ & $1,57 E+03$ & 1,28 \\
MUON+ & $4,48 E+01$ & $4,52 E+01$ & 0,89 \\
MUON- & $4,18 E+03$ & $4,43 E+03$ & 5,81 \\
PION+ & $3,32 E+03$ & $3,27 E+03$ & 1,52 \\
PION- & $3,22 E+03$ & $3,20 E+03$ & 0,62 \\
KAON+ & $2,47 E+01$ & $2,18 E+01$ & 12,5 \\
KAON- & $1,24 E+01$ & $1,39 E+01$ & 11,4 \\
LAMBDA & $6,10 E-01$ & $3,83 E-01$ & 45,72 \\
ALAMBDA & $3,95 E-03$ & $7,26 E-03$ & 59,05 \\
SIGMA- & $1,12 E-03$ & $2,99 E-03$ & 91,00 \\
SIGMA+ & $2,51 E-03$ & $3,24 E-03$ & 25,39 \\
KAONZERO & $2,97 E+01$ & $3,43 E+01$ & 14,38 \\
AKAONZER & $3,82 E+01$ & $4,33 E+01$ & 12,52 \\
ASIGMA- & $1,51 E-04$ & $2,09 E-04$ & 32,22 \\
ASIGMA+ & $2,75 E-04$ & $1,71 E-04$ & 46,64 \\
XSIZERO & $1,18 E-04$ & $4,48 E-04$ & 116,61 \\
AXSIZERO & $1,09 E-04$ & $1,88 E-04$ & 53,20 \\
AXSI+ & $1,25 E-04$ & & \\
\hline & & &
\end{tabular}


TABLE 4: Number of secondaries generated in inelastic interactions.

\begin{tabular}{|c|c|c|c|}
\hline \multirow[b]{2}{*}{ Particle name } & \multicolumn{3}{|c|}{ Number of particle } \\
\hline & Atmosphere & Kaidun & $\begin{array}{c}\text { Relative } \\
\text { difference (\%) }\end{array}$ \\
\hline 4-HELIUM & $1,80 E+06$ & $1,82 E+06$ & 1,10 \\
\hline 3-HELIUM & $1,31 E+05$ & $1,33 E+05$ & 1,52 \\
\hline TRITON & $1,30 E+05$ & $1,31 E+05$ & 0,77 \\
\hline DEUTERON & $3,00 E+05$ & $3,02 E+05$ & 0,66 \\
\hline PROTON & $2,41 E+06$ & $2,44 E+06$ & 1,24 \\
\hline APROTON & $1,44 E+03$ & $1,47 E+03$ & 2,06 \\
\hline ELECTRON & $1,38 E+02$ & $1,35 E+02$ & 2,20 \\
\hline POSITRON & $1,38 E+02$ & $1,34 E+02$ & 2,94 \\
\hline NEUTRIE & $2,89 E-02$ & $7,12 E-02$ & 84,52 \\
\hline ANEUTRIE & $4,89 E-01$ & $9,96 E-01$ & 68,28 \\
\hline PHOTON & $7,90 E+05$ & $8,03 E+05$ & 1,63 \\
\hline NEUTRON & $3,09 E+06$ & $3,13 E+06$ & 1,29 \\
\hline ANEUTRON & $1,44 E+03$ & $1,46 E+03$ & 1,38 \\
\hline MUON+ & $5,68 E+01$ & $5,16 E+01$ & 9,59 \\
\hline MUON- & $4,26 E+01$ & $4,12 E+01$ & 3,34 \\
\hline KAONLONG & $2,04 E+02$ & $1,74 E+02$ & 15,87 \\
\hline PION+ & $2,92 E+05$ & $2,94 E+05$ & 0,68 \\
\hline PION- & $2,77 E+05$ & $2,79 E+05$ & 0,72 \\
\hline $\mathrm{KAON}+$ & $1,19 E+04$ & $1,20 E+04$ & 0,84 \\
\hline KAON- & $4,32 E+03$ & $4,37 E+03$ & 1,15 \\
\hline LAMBDA & $6,70 E+03$ & $6,86 E+03$ & 2,36 \\
\hline ALAMBDA & $1,41 E+02$ & $1,39 E+02$ & 1,43 \\
\hline KAONSHRT & $2,03 E+02$ & $1,79 E+02$ & 12,57 \\
\hline SIGMA- & $2,01 E+03$ & $1,98 E+03$ & 1,50 \\
\hline SIGMA+ & $2,35 E+03$ & $2,34 E+03$ & 0,43 \\
\hline SIGMAZER & $3,55 E+03$ & $3,53 E+03$ & 0,56 \\
\hline PIZERO & $3,64 E+05$ & $3,67 E+05$ & 0,82 \\
\hline KAONZERO & $1,14 E+04$ & $1,14 E+04$ & - \\
\hline AKAONZER & $4,11 E+03$ & $4,16 E+03$ & 1,21 \\
\hline NEUTRIM & $4,15 E+03$ & $4,39 E+03$ & 5,62 \\
\hline ANEUTRIM & $2,97 E-01$ & $3,08 E-01$ & 3,64 \\
\hline ASIGMA- & $4,62 E+01$ & $4,46 E+01$ & 3,52 \\
\hline ASIGMAZE & $4,26 E+01$ & $4,25 E+01$ & 0,24 \\
\hline ASIGMA+ & $4,15 E+01$ & $4,68 E+01$ & 12,00 \\
\hline XSIZERO & $1,28 E+02$ & $1,26 E+02$ & 1,57 \\
\hline AXSIZERO & $8,77 E+00$ & $1,08 E+01$ & 20,75 \\
\hline XSI- & $1,09 E+02$ & $1,09 E+02$ & - \\
\hline AXSI+ & $8,21 E+00$ & $8,82 E+00$ & 7,16 \\
\hline OMEGA- & $3,93 E-01$ & $6,92 E-02$ & 140,11 \\
\hline AOMEGA+ & $8,51 E-01$ & $1,10 E+00$ & 25,53 \\
\hline $\mathrm{D}+$ & $1,34 E-04$ & $2,40 E-03$ & 178,85 \\
\hline D- & $2,29 E-04$ & $2,35 E-04$ & 2,59 \\
\hline D0 & $6,17 E-04$ & $3,45 E-04$ & 56,55 \\
\hline DOBAR & $3,51 E-04$ & $2,98 E-03$ & 157,85 \\
\hline DS+ & $6,74 E-05$ & $1,45 E-04$ & 73,07 \\
\hline DS- & $2,54 E-04$ & $8,29 E-05$ & 101,57 \\
\hline LAMBDAC+ & $1,34 E-04$ & $3,17 E-04$ & 81,15 \\
\hline NEUTRIT & & $2,30 E-03$ & \\
\hline ANEUTRIT & & $2,30 E-03$ & \\
\hline OMEGAC0 & & $9,17 E-05$ & \\
\hline
\end{tabular}

TABLE 5: Decay products generated in inelastic interactions.

\begin{tabular}{|c|c|c|c|}
\hline \multirow[b]{2}{*}{ Particle name } & \multicolumn{3}{|c|}{ Number of particle } \\
\hline & Atmosphere & Kaidun & $\begin{array}{c}\text { Relative } \\
\text { difference (\%) }\end{array}$ \\
\hline PROTON & $7,96 E+03$ & $8,05 E+03$ & 1,12 \\
\hline APROTON & $1,55 E+02$ & $1,52 E+02$ & 1,95 \\
\hline ELECTRON & $2,92 E+05$ & $2,94 E+05$ & 0,68 \\
\hline POSITRON & $3,10 E+05$ & $3,12 E+05$ & 0,64 \\
\hline NEUTRIE & $3,05 E+05$ & $3,07 E+05$ & 0,65 \\
\hline ANEUTRIE & $2,87 E+05$ & $2,89 E+05$ & 0,69 \\
\hline PHOTON & $7,70 E+05$ & $7,75 E+05$ & 0,65 \\
\hline NEUTRON & $6,90 E+03$ & $6,89 E+03$ & 0,15 \\
\hline ANEUTRON & $1,34 E+02$ & $1,41 E+02$ & 5,09 \\
\hline MUON+ & $3,13 E+05$ & $3,15 E+05$ & 0,64 \\
\hline MUON- & $2,97 E+05$ & $3,00 E+05$ & 1,01 \\
\hline PION+ & $1,48 E+04$ & $1,47 E+04$ & 0,68 \\
\hline PION- & $2,01 E+04$ & $2,02 E+04$ & 0,50 \\
\hline $\mathrm{KAON}+$ & $5,43 E-01$ & $7,37 E-01$ & 30,31 \\
\hline KAON- & $2,30 E-01$ & $4,76 E-02$ & 131,41 \\
\hline LAMBDA & $3,79 E+03$ & $3,76 E+03$ & 0,79 \\
\hline ALAMBDA & $6,04 E+01$ & $6,32 E+01$ & 4,53 \\
\hline SIGMA+ & $6,47 E-05$ & - & \\
\hline PIZERO & $2,16 E+04$ & $2,14 E+04$ & 0,93 \\
\hline KAONZERO & $2,33 E-04$ & $5,30 E-04$ & 77,85 \\
\hline AKAONZER & $4,09 E-04$ & $7,94 E-04$ & 64,01 \\
\hline NEUTRIM & $5,98 E+05$ & $6,02 E+05$ & 0,66 \\
\hline ANEUTRIM & $6,01 E+05$ & $6,05 E+05$ & 0,66 \\
\hline XSIZERO & $1,64 E-01$ & $8,05 E-04$ & 198,05 \\
\hline AXSIZERO & $3,08 E-01$ & $1,96 E-01$ & 44,44 \\
\hline AXSI+ & $1,54 E-04$ & $1,67 E-01$ & 199,63 \\
\hline KAONLONG & - & $5,74 E-05$ & \\
\hline KAONSHRT & - & $5,74 E-05$ & \\
\hline XSI- & - & $2,35 E-02$ & \\
\hline TAU- & - & $8,29 E-05$ & \\
\hline NEUTRIT & - & $8,29 E-05$ & \\
\hline ANEUTRIT & - & $8,29 E-05$ & \\
\hline
\end{tabular}

interactions. Also, differences in the number of particles produced from atmosphere and Kaidun interactions are outstanding. For example, tau neutrinos which do not occur after CRs-atmosphere interactions have been observed after CRs-Kaidun interactions. Given results as a result of this paper may be useful for space and particle researchers.

\section{References}

[1] A. V. Ivanov, "Is the Kaidun meteorite a sample from Phobos?" Solar System Research, vol. 38, no. 2, pp. 97-107, 2004.

[2] A. V. Ivanov, N. N. Kononkova, S. V. Yang, and M. E. Zolensky, "The Kaidun meteorite: clasts of alkaline-rich fractionated 
materials," Meteoritics and Planetary Science, vol. 38, no. 5, pp. 725-737, 2003.

[3] A. V. Ivanov, N. N. Kononkova, and M. E. Zolensky, "Pegmatoid objects in a sample of the Kaidun meteorite," Geochemistry International, vol. 46, no. 8, pp. 759-774, 2008.

[4] Y. A. Shukolyukov and A. V. Ivanov, "Noble gases in the unique Kaidun meteorite,” Petrology, vol. 15, no. 4, pp. 408-425, 2007.

[5] M. Petitat, Y. Marrocchi, K. D. Mckeegan et al., " $53 \mathrm{Mn}-53 \mathrm{Cr}$ ages of Kaidun carbonates," Meteoritics and Planetary Science, vol. 46, no. 2, pp. 275-283, 2011.

[6] F. Brandstaetter and A. Ivanov, "On the occurrence of cubanite in the Kaidun meteorite," Meteoritics and Planetary Science, vol. 46, article A28, 2011.

[7] A. V. Fisenko, L. F. Semjonova, A. S. Aronin, L. N. Bol'sheva, and A. V. Ivanov, "THE Kaidun meteorite: colloidal diamonds from crandci clasts," Meteoritics and Planetary Science, vol. 34, article A35, 1999.

[8] A. V. Fisenko, A. B. Verkhovsky, L. F. Semenova, A. V. Ivanov, and C. T. Pillinger, "The Kaidun meteorite: interstellar diamond in the CR and CI carbonaceous components," Geochemistry International, vol. 38, no. 3, pp. S294-S301, 2000.

[9] L. F. Migdisova, A. V. Ivanov, N. N. Kononkova, F. Brandstaetter, and G. Kurat, "The Kaidun meteorite: a fragment of a highcalcium primitive achondrite," Geochemistry International, vol. 38, no. 3, pp. S369-S374, 2000.

[10] A. V. Ivanov, M. E. Zolensky, N. N. Kononkova, S. V. Yang, and I. A. Stroganov, "Kaidun meteorite: crystals of oxides in cavities," Geochemistry International, vol. 44, no. 3, pp. 249-257, 2006.

[11] A. V. Ivanov, M. A. Ivanova, and N. N. Kononkova, "Concentrically zonal textures in a sample of the Kaidun meteorite," Geochemistry International, vol. 45, no. 10, pp. 957-970, 2007.

[12] G. J. MacPherson, D. W. Mittlefehldt, M. E. Lipschutz et al., "The Kaidun chondrite breccia: petrology, oxygen isotopes, and trace element abundances," Geochimica et Cosmochimica Acta, vol. 73, no. 18, pp. 5493-5511, 2009.

[13] A. V. Ivanov, D. D. Badyukov, and N. N. Kononkova, "Kaidun meteorite: a fragment of crystalline rock from a new parent body," Geochemistry International, vol. 48, no. 9, pp. 862-870, 2010.

[14] A. A. Yaroshevsky and A. V. Ivanov, "Geochemical diversity of meteorite minerals: systematics and nomenclature of phosphides and silicides," Geochemistry International, vol. 48, no. 8 , pp. 808-814, 2010.

[15] K. Ziegler, A. N. Krot, A. V. Ivanov, M. A. Ivanova, and E. D. Young, "Oxygen isotope compositions of differentiated fragments from Kaidun," Meteoritics and Planetary Science, vol. 47, no. S1, article A431, 2012.

[16] D. Harries, F. Langenhorst, and M. E. Zolensky, "Sulfide formation in cm chondrites: nebula vs. Parent body processes," Meteoritics and Planetary Science, vol. 47, no. S1, article A173, 2012.

[17] E. Görmez and T. Korkut, "FLUKA Monte Carlo simulations on neutron interactions with FeCrP and FeTiP," Physicochemichal Problems of Mineral Processing, vol. 49, no. 2, pp. 453-462, 2013.

[18] G. Kurat, E. Zinner, F. Brandstätter, and A. V. Ivanov, "Enstatite aggregates with niningerite, heideite, and oldhamite from the Kaidun carbonaceous chondrite: relatives of aubrites and $\mathrm{EH}$ chondrites?" Meteoritics and Planetary Science, vol. 39, no. 1, pp. 53-60, 2004.

[19] M. E. Zolensky, M. Gounelle, T. Mikouchi et al., "Andreyivanovite: a second new phosphide from the Kaidun meteorite," American Mineralogist, vol. 93, no. 8-9, pp. 1295-1299, 2008.
[20] A. V. Ivanov, M. E. Zolensky, and S. V. Yang, "The Kaidun meteorite: fast crystallization of a fragment from a superheated melt," Meteoritics and Planetary Science, vol. 35, no. S5, article A82, 2000.

[21] A. V. Ivanov, M. E. Zolensky, S. V. Yang, and A. A. Ariskin, "The Kaidun meteorite: evidence for aqueous alteration and precipitation," Meteoritics and Planetary Science, vol. 35, no. S5, article A82, 2000.

[22] L. L. Kashkarov, N. N. Korotkova, A. Y. Skripnik, and K. I. Ignatenko, "Radiation-thermal history of Kaidun meteorite on data of track study in silicate mineral crystals and glass fragment," Geokhimiya, vol. 10, p. 1409, 1995.

[23] M. Zolensky and A. Ivanov, "The Kaidun microbreccia meteorite: a harvest from the inner and outer asteroid belt," Chemie der Erde, vol. 63, no. 3, pp. 185-246, 2003.

[24] M. A. Ivanova, N. N. Kononkova, and A. V. Ivanov, "First recorded find of ordinary chondrite material in the Kaidun meteorite," Solar System Research, vol. 39, no. 2, pp. 150-156, 2005.

[25] M. A. Ivanova, N. N. Kononkova, and A. V. Ivanov, "Concentriczoned inclusions in the Kaidun meteorite," Meteoritics and Planetary Science, vol. 41, no. S8, article A85, 2006.

[26] G. Bellini, J. Benzigerb, D. Bick et al., "Cosmogenic Backgrounds in Borexino at $3800 \mathrm{~m}$ water-equivalent depth," Journal of Cosmology and Astroparticle Physics, vol. 2013, no. 8, article 049, 2013.

[27] T. C. Slaba, S. R. Blattnig, B. Reddell, A. Bahadori, R. B. Norman, and F. F. Badavi, "Pion and electromagnetic contribution to dose: comparisons of HZETRN to Monte Carlo results and ISS data," Advances in Space Research, vol. 52, no. 1, pp. 62-78, 2013.

[28] Z. W. Lin, J. H. Adams, A. F. Barghouty et al., "Comparisons of several transport models in their predictions in typical space radiation environments," Advances in Space Research, vol. 49, no. 4, pp. 797-806, 2012.

[29] L. Sihver, M. Lantz, M. Takechi et al., "A comparison of total reaction cross section models used in particle and heavy ion transport codes," Advances in Space Research, vol. 49, no. 4, pp. 812-819, 2012.

[30] A. Ferrari, P. R. Sala, A. Fasso, and J. Ranft, "FLUKA: a multiparticle transport code," CERN-2005-10, INFN/TC_05/11, SLAC-R-773, 2005.

[31] T. Korkut, A. Karabulut, G. Budak, B. Aygün, O. Gencel, and A. Hançerlioĝullari;, "Investigation of neutron shielding properties depending on number of boron atoms for colemanite, ulexite and tincal ores by experiments and FLUKA Monte Carlo simulations," Applied Radiation and Isotopes, vol. 70, no. 1, pp. 341-345, 2012.

[32] S. Roesler, R. Engel, and J. Ranft, "The Monte Carlo event generator DPMJET-III," in Proceedings of the Monte Carlo Conference, Springer, Lisbon, Portugal, October 2000.

[33] G. Battistoni, A. Margiotta, S. Muraro, and M. Sioli, "FLUKA as a new high energy cosmic ray generator," Nuclear Instruments and Methods in Physics Research A, vol. 626-627, pp. S191-S192, 2011. 

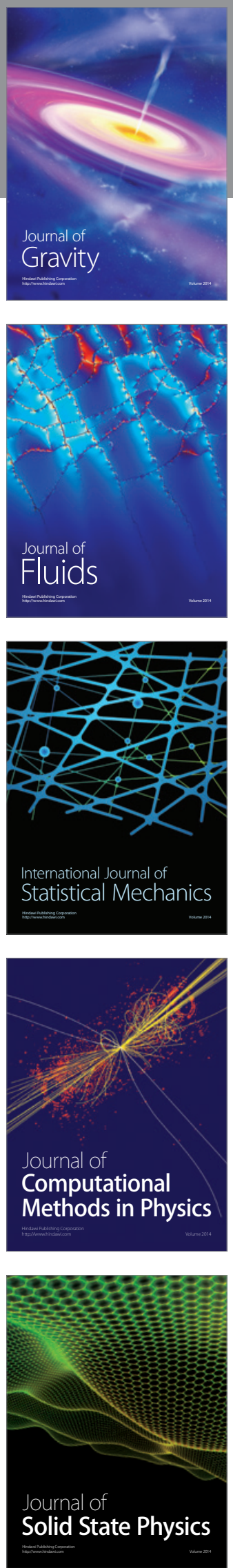

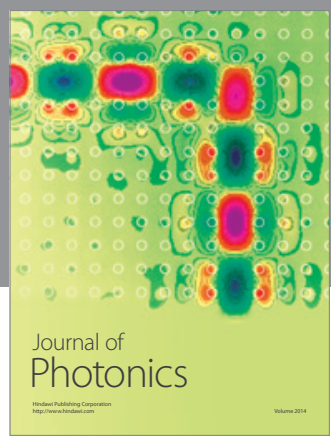

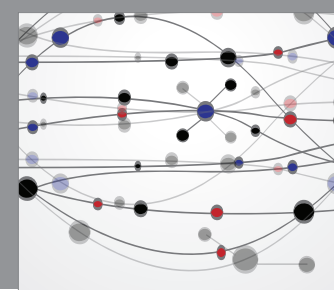

The Scientific World Journal

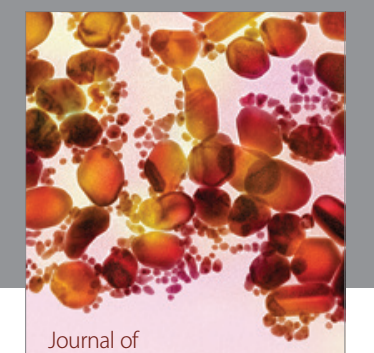

Soft Matter
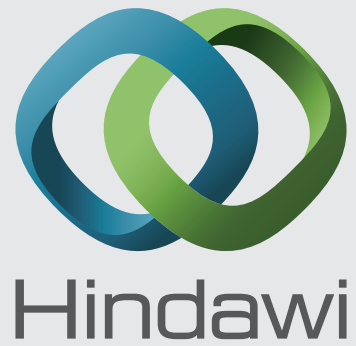

Submit your manuscripts at

http://www.hindawi.com
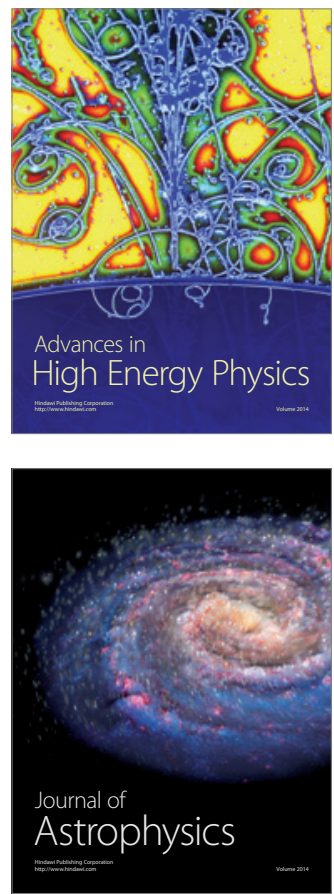
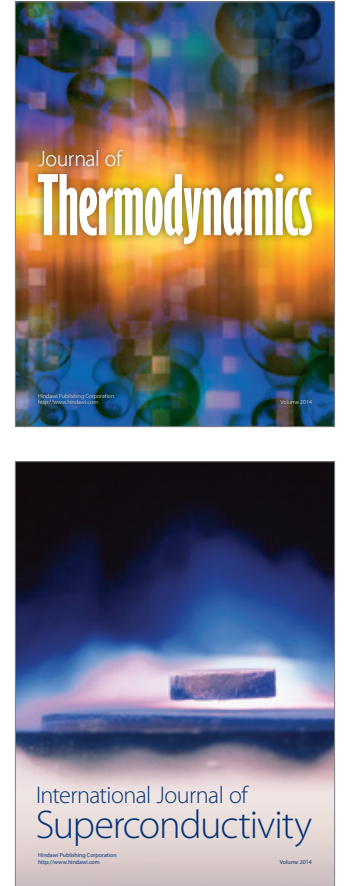
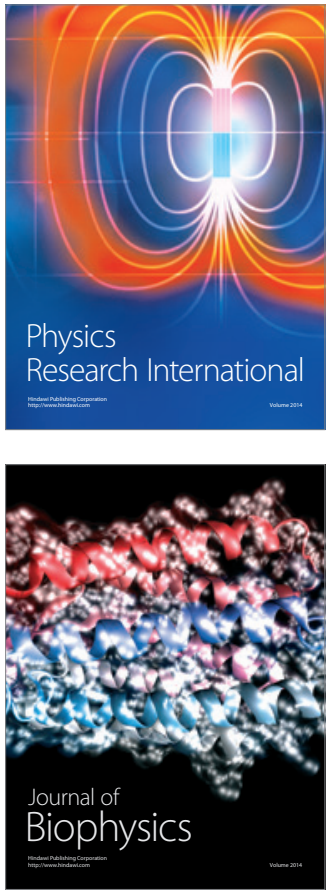
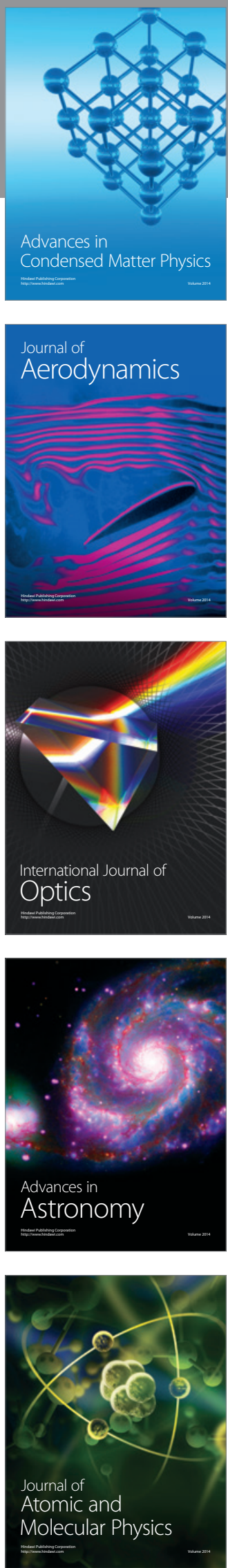